\title{
Label-free and Antibody-free Protein Detection Based on Organic Thin-Film Transistors
}

\author{
Tsuyoshi Minami \\ Institute of Industrial Science, the University of Tokyo, 4-6-1 Komaba, Meguro-ku, Tokyo 153-8505, Japan \\ tminami@iis.u-tokyo.ac.jp
}

\begin{abstract}
:
A label-free and antibody-free detection of proteins has been achieved using an organic thin-film transistor (OTFT)-based sensor functionalized with artificial receptors. The OTFT responds to protein recognition behavior of the receptor. The demonstration of the protein detection could pave the way to the development of disposable and portable bio-sensing systems.
\end{abstract}

Key words: Organic thin-film transistors, Artificial receptors, Self-assembled monolayers

\section{Introduction}

Development of analytical methods for biomarkers such as proteins has great significance in the fields of clinical diagnostics, food safety tests, proteomics, etc. Although the features (a high sensitivity and reliability) of conventional assays are useful for the accurate detection of proteins, the assays have difficulty in the application to on-site detection at various situations for the following reasons. 1) Labelling processes for analytes are complicated. 2) It takes time for users to acquire analysis results, because pre-treatments of immune proteins are timeconsuming processes. 3) For quantitative analysis, relatively large equipment and expensive reagents are required; for instance, spectroscopic apparatus and naturally derived substances are generally essential to optical-based assays such as enzymelinked immunosorbent assays (ELISAs). Therefore, compact, low-cost, and easy methods for protein analyses are highly desired.

To solve the above problems, micro-total analysis systems $(\mu T A S)$ toward the on-site detection of biomarkers have been demonstrated in the fields of analytical chemistry and device engineering. Especially, microfluidic technology of $\mu$ TAS is a useful approach for reducing the total measurement time of proteins. In those devices, various transducing mechanisms for detection-signal of proteins can be employed. For example, organic thinfilm transistor (OTFT)-based sensing platforms for several biomarkers have been demonstrated in recent years. OTFTs can be easily fabricated on elastic substrates using wet processes. These electrical switchers are not only valuable transducers for chemical/bio-sensors, but also the prospective devices for flexible displays, etc. Thus, the components of the sensing system could be integrated into a single-chip using OTFT-based circuits. To date, OTFT-based sensors usually rely on the above-mentioned immunoassays, while the development of the detection system using artificial receptors in the field of OTFT devices is still in its early stage. In this study, we herein propose the design and fabrication strategy to achieve easy-touse sensor devices on the basis of an OTFT modified with self-assembled monolayers (SAMs) of the coordination-bonding-based receptors for target proteins (=phosphoprotein and histidine-rich protein) (Fig. 1). Coordination-bonding-based artificial receptors are often employed for optical protein detection, because they are not requiring bio-derived materials and labelling processes. For these reasons, we decided to develop a label-free and antibody-free sensor devices based on OTFTs modified with the artificial protein receptors such as zinc(II)dipicolylamine ( $\mathrm{Zn}$ "-dpa) or nickel(II)-nitrilotiracetic acid (Nill-nta).

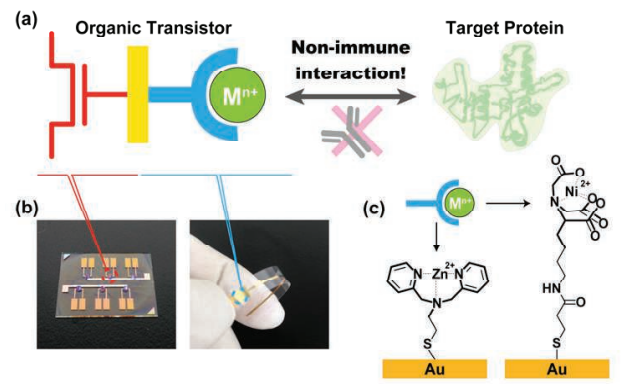

Fig. 1 (a) Schematic illustration of the label-free and antibody-free sensor device based on the OTFT. (b) Photographs show the fabricated sensing devices. (c) Sensing electrodes are functionalized with $\mathrm{Zn}^{\prime \prime}$ dpa (left) or Nil'-nta (right) complex.

\section{Experimental}


The label-free protein sensor based on the OTFT was designed and fabricated as follows (Fig. 2). To apply the OTFT for accurate detection of proteins, it is necessary to stabilize electrical signals of the fabricated device. Toward this end, the OTFT was constructed with a conjugated polymer (PBTTT)based active layer, and a SAM (=tetradecylphosphonic acid, $\mathrm{C}_{14}$-PA)-based dielectric layer which provides high electric capacity. Furthermore, it provides possible to take an accurate measurement of output signals since the transducer ( $=$ the OTFT) is separated from the detection portion (= the extended-gate), which can prevent degradation of the active layer by water. The chemical functionalization of the gold extended-gate electrode toward recognition of the target proteins was carried with the SAM of artificial receptors.

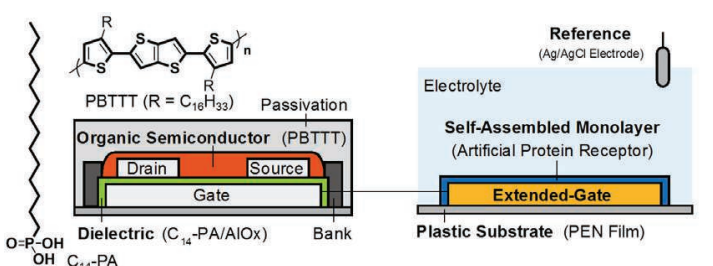

Fig. 2 Schematic structure of the designed extended-gate type OTFT.

\section{Results and Discussion}

Firstly, we attempted the titration experiment of the fabricated OTFT sensor by using phosphoprotein (= $\alpha$-casein) [1]. Although $\alpha$-casein is a good protein source, it is also known as an allergen which is presumed to bind human IgE or IgG. Hence, the detection of $\alpha$-casein is important in food processing. As shown in Fig. 3(a), the output current of the OTFT device was significantly decreased upon addition of increased amounts of $\alpha$-casein. The $80 \%$ and $90 \%$ response times for the addition of $\alpha$-casein were within 1 and $3 \mathrm{~min}$, respectively (Fig. 3(b)). The limit of detection (LOD) of the developed sensor for $\alpha$ casein was estimated to be $0.22 \mu \mathrm{g} \mathrm{mL}^{-1}$, which is lower than that of the previously reported a fluorescent probe based on a $\mathrm{Zn}$ "-dpa receptor. To confirm whether the observed response stemmed

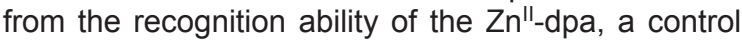
titration experiment was carried out (Fig. 3 (c)). In the case of dephospho- $\alpha$-casein, the current response of the OTFT was weaker than that of phospho- $\alpha$-casein. While weaker, a certain response to dephospho- $\alpha$ casein is attributed to the residual phosphorylated sites in the protein. In addition, weak or almost no response to $\beta$-galactosidase, albumin, and lysozyme was observed. These results support the claim that the sensing properties originate from the recognition ability of the $\mathrm{Zn}^{\text {"l-}}$-dpa.

Next, we performed the electrical detection of bovine serum albumin (BSA) utilizing the Ni"-nta-modified OTFT [2]. An abnormal lowering of albumin concentration in blood is caused by many diseases such as liver disease, nephrotic syndrome, malnutrition, etc. In this regard, the detection of the albumin level is important for diagnosis. As a result, an electrical titration experiment revealed a distinct decrease in the output current (IDS) of the OTFT upon addition of BSA in a HEPES buffer solution (Fig. 4
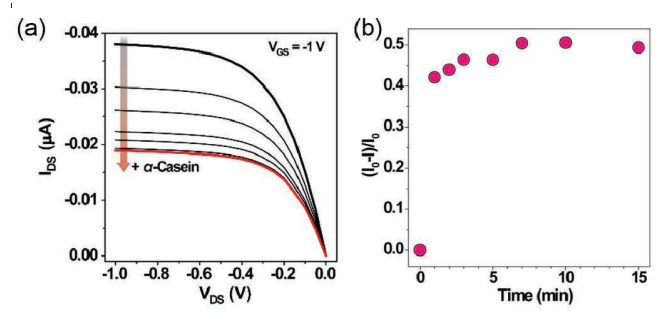

(c)

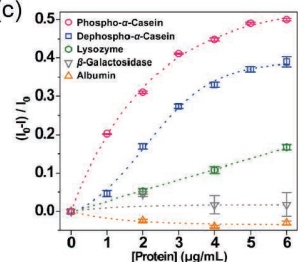

Fig. 3 (a) Output characteristics of the OTFT upon addition of increased amounts of $\alpha$-casein. [ $\alpha$ Casein] = 0-6 $\mu \mathrm{g} \mathrm{mL}^{-1}$. (b) Time dependency of the electrical response to $\alpha$-casein $\left(6 \mu g \mathrm{~mL}^{-1}\right)$. (c) Changes in the output current $(n=3)$ of the OTFT device by proteins at various concentration.

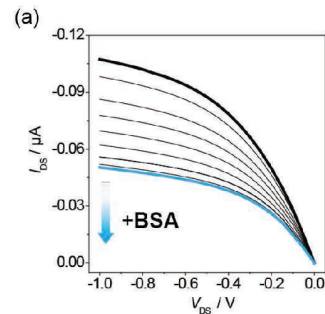

(b)

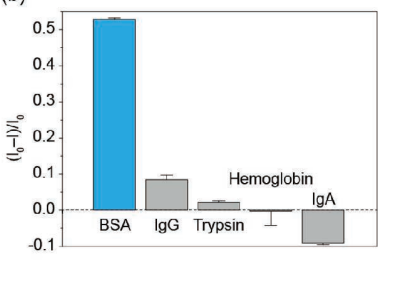

Fig. 4 (a) The output characteristics of the OTFT upon the addition of BSA. [BSA] $=0-10 \mathrm{ng} \mathrm{mL^{-1 }}$. (b) Changes in the output current $(n=3)$ of the OTFT sensor by proteins. [Protein] $=10 \mathrm{ng} \mathrm{mL} \mathrm{L}^{-1}$.

(a)). The estimated LOD for BSA was $40 \mathrm{pg} \mathrm{mL}^{-1}$. Notably, it is comparable to, or lower than, those of recently reported immunoassays or luminescent probes for BSA. We also investigated the protein selectivity of the Nill-nta-modified OTFT sensor (Fig. 4(b)). As a result, the electrical response of the OTFT to BSA was obviously high, whereas those for IgG, $\operatorname{lgA}$, trypsin, and hemoglobin were very weak. Because control analytes have no consecutive histidine residues, the observed selective response to BSA indicate that the binding affinity between the $\mathrm{Ni}^{\prime \prime}-$ nta on the extended-gate electrode and the histidine residue sites of BSA is the prime influence for the changes in the drain current.

In summary, we have successfully demonstrated the label-free and antibody-free electrical sensing of the target proteins using extended-gate type OTFT devices. We believe that our preliminary results on the OTFT-based protein sensors could pave the way to a new approach for disposable electronic devices toward on-site detection of various analytes.

\section{References}

[1] T. Minamiki, T. Minami, P. Koutník, P. Anzenbacher, Jr, S. Tokito, Anal. Chem. 88, 1092 (2016); doi: 10.1021/acs.analchem.5b04618

[2] T. Minamiki, Y. Sasaki, S. Tokito, T. Minami, ChemistryOpen 6, 472 (2017); doi: 10.1002/open.201700070 